\title{
What is the impact of continuous cover forestry compared to clearcut forestry on stand-level biodiversity in boreal and temperate forests? A systematic review protocol
}

\author{
Meelis Seedre* $\mathbb{C}^{*}$, Adam Felton and Matts Lindbladh
}

\begin{abstract}
Background: The ecosystem services provided by forests are essential for societal well-being. Production forests are increasingly expected to provide a range of ecosystem services in addition to wood biomass, as well as the biodiversity upon which many of these services depend. Production forests can be managed using different methods that affect the habitat provided and the biodiversity supported. Clearcutting (CC) is a widely used forest management system that has been criticised due to its negative effects on biodiversity. Alternative less intensive forest management systems have been developed with the hope of producing comparable levels of biomass with fewer negative impacts on forest biodiversity. One of these alternatives is continuous cover forestry (CCF); a management system that always maintains tree cover in an uneven-aged production forest stand. Many studies have been conducted which contrast the effects of CCF and CC on biodiversity with varying results. The aim of the review is to explore how CCF system compares to CC in terms of outcomes for terrestrial forest biodiversity.

Methods: Due to the diverse vocabulary used to describe CCF, a systematic search for terms was carried out and a comprehensive search string will be used to maximise the likelihood of finding all relevant papers. We will gather, summarise and synthesise primary field studies, both peer-reviewed and grey literature, from temperate and boreal forest comparing biodiversity in CCF and CC stands. Species richness and abundance of plants, animals and fungi will be used to conduct a meta-analysis. Other biodiversity indicators and indices will be used for a narrative synthesis. As the effects of forest management depend on local conditions, we place a special emphasis on exploring the influence of various effect modifiers.
\end{abstract}

Keywords: Alternative forest management, Even-aged silviculture, Uneven-aged silviculture, Partial harvest, Selection harvest, Clear cut, Clear fell

\footnotetext{
*Correspondence: Meelis.Seedre@slu.se

Southern Swedish Forest Research Centre, The Swedish University

of Agricultural Sciences, P.O. Box 49, 23053 Alnarp, Sweden
} 


\section{Background}

Forests provide ecosystem services that are essential for human well-being $[1,2]$ and sustainable forest management is the cornerstone of the maintenance of these services. Production forests are increasingly expected to sustain a diverse array of these services and the biodiversity upon which many services depend $[3,4]$. Nevertheless, the focus of management has not been balanced in terms of service provision, and changes to management may be necessary to improve the provision of forest ecosystems and the services they provide.

Forests can be managed with varying intensity using different methods that affect forest conditions and the biodiversity supported. One common intensive management system is clear-cut (CC) forestry. Rotational CC systems follow a cyclic pattern of harvesting most or all trees in a mature forest stand (i.e. a forest management unit), and subsequent regeneration (often planting), resulting in a structurally homogeneous even-aged forest. The widespread use of this system has been criticised due to its negative effects on biodiversity $[3,5,6]$. Therefore, alternatives to clearcutting are often advocated [7, 8]. One common alternative management system is retention forestry, where typically $2-30 \%$ of trees are retained after the cut, and in the case of shelterwood and clear-cut free forestry systems, harvested when regenerating trees have sufficiently established. Effects of retention forestry on biodiversity are summarised in two recent meta-analyses $[9,10]$. Both of those studies find that retention forestry can have positive effects on biodiversity. However, in stands managed with this method, the retained trees will gradually die, and the forest will eventually become even-aged with some structural heterogeneity provided by the old dead trees.

Continuous cover forestry (CCF) is another silvicultural method that differs from $\mathrm{CC}$ and shelterwood systems, in that it always maintains tree cover and heterogeneous forest structure. This might have additional benefits to biodiversity compared to retention forestry. Definitions of CCF vary [11], and here we define CCF as a silvicultural method involving the partial harvest of production stems, which always maintains at least 30\% tree cover (by e.g. basal area, volume, etc.), and at least two-age classes of production trees, and thereby, an uneven-aged forest structure. This definition captures the essence of CCF and importantly, excludes studies dealing with retention, shelterwood and even-aged forestry.

Whereas the disturbance associated with CC is comparable to large-scale stand-replacing natural disturbances, CCF better mimics the effect of smaller non-stand replacing disturbances (i.e. a partial disturbance that does not kill all trees in a stand) and their effects [5, 12]. Due to these differences, CCF is often assumed to be better than
$\mathrm{CC}$ for maintaining forest biodiversity and a range of ecosystem services [7]. Biodiversity refers to the encompassing variability among all living organisms, including diversity within species, between species, and of ecosystems [13]. In this study, we focus on those studies which contrast the implications of CCF and CC on the species richness and abundance of three key elements of terrestrial biodiversity, namely plants, animals and fungi. CCF is currently being considered as an alternative to CC in several northern European countries, including Sweden. As our focal concern is to understand the potential implications for biodiversity from converting even-aged stands to CCF within this region, we limit our review to those studies conducted in the temperate and boreal regions.

A recent non-systematic review concluded that uneven-aged silviculture is not better than even-aged silviculture at maintaining biodiversity [14]. Likewise, a study comparing these management systems in European beech forest across distinct spatial scales found that the availability of different aged stands in the landscape is more beneficial to biodiversity than high stand-level heterogeneity [15]. In contrast, a recent modelling study found that CCF should provide for higher biodiversity than CC [16], and a qualitative review conducted for the Fennoscandia region found that CCF better retained many late-successional forest species than CC [7]. To date, systematic qualitative and meta-analysis approaches have not been used to contrast biodiversity in CCF and CC systems. This significantly hinders evidence-based decision making in light of the varying results provided by different studies.

\section{Objectives of the review}

The primary aim of the proposed review is to determine how CCF and CC stands contrast in terms of their implications for the plant, animal and fungal species elements of terrestrial stand-level forest biodiversity. To estimate management effect on biodiversity, we will focus on most commonly reported biodiversity indicators, namely species richness and abundance, but also consider other relevant metrics when possible. How CCF and CC management systems influence biodiversity is likely to be influenced by multiple context-dependent factors (e.g. forest type, geographical location, time since treatment, landscape context). Therefore, assessing the influence of these effect modifiers will be prioritised.

\section{Primary questions}

What is the impact of continuous cover forestry compared to clearcut forestry on stand-level biodiversity in boreal and temperate forests? 


\section{Question components}

Population: Boreal and temperate forest.

Interventions: $\mathrm{CCF}$ approaches.

Comparator: Clearcutting.

Outcomes: Biodiversity of terrestrial plants, animals and fungi represented by measures of taxonomic diversity; species richness and abundance for meta-analysis and other diversity measures (e.g. community composition, diversity indexes, the presence of forest interior or rare species) for narrative synthesis.

\section{Methods}

\section{Searching for articles}

We will follow the guidelines for systematic reviews in environmental management V.5.0, issued by the collaboration for environmental evidence [17]. We have also supplemented the protocol with a ROSES form (Additional file 1) [18]. We will use a comprehensive search strategy and search for relevant literature from a diverse and broad array of bibliographic databases.

1. CABI database of forest science (http://www.cabi. org/forestscience/).

2. Directory of Open Access Journals (https://doaj. $\operatorname{org} /)$.

3. OpenGrey (http://www.opengrey.eu).

4. PQDT Open (https://pqdtopen.proquest.com/).

5. Scopus (https://www.scopus.com/).

6. Web of Science Core Collection (http://apps.webof knowledge.com).

7. Zenodo (https://zenodo.org).

We will also search for articles using two search engines, Google Scholar (https://scholar.google.com) and BASE (https://www.base-search.net). Furthermore, we will search for articles in a specialist website, U.S. Forest Service (https://www.fs.usda.gov).

The scoping work revealed that relatively few studies can be found when specific terms relevant to the study question components are used. This stemmed from the inconsistent use of vocabulary among researchers when describing CCF and associated harvesting methods, rather than a lack of relevant literature. This problem has likely caused researchers and other interested parties to underestimate the amount of research carried out on this topic. We therefore performed a systematic search of terms related to CCF and associated harvesting methods. We systematically extracted all relevant terms used in papers that have reviewed the terminology $[7,11,19$, 20]. This resulted in 64 unique terms (Additional file 2). Most of those terms consisted of several words, the first word indicating a cutting or management type and the last word specifying that it refers to forestry (i.e. selection cutting, selection harvesting, selection felling, etc.). To shorten and simplify the search string we individually tested different terms and removed terms that provided no additional results. Some general terms (e.g. alternative management, nature-oriented, ecosystem management, etc.) resulted in a large number of articles without relevance to the topic addressed. We supplemented these terms with forest management specific terms (e.g. alternative silviculture, alternative harvest, etc.) to help ensure relevant literature was not excluded. This resulted in total 78 terms for the intervention. For the same reason, we will limit our search to forest relevant subject categories in Web of Science (WoS) and research areas in Scopus (Table 1). We will then supplemented these terms with terms relevant to the comparator, i.e. clearcut.

We will not limit the search to the boreal or temperate forest (population) to ensure that relevant studies will not be excluded. We will also not limit the search to biodiversity relevant studies (outcome) since it is very difficult to have an all-inclusive biodiversity search string. This approach increases the workload but maximises the chances of finding all relevant biodiversity studies. We will search within titles, abstracts and keywords. After screening, newly discovered terms will be used to perform another search. To assess the comprehensiveness of the search, we will use the following articles as benchmarks: $[7,11,14,15,19]$. We will use the following search string.

\begin{tabular}{|c|c|c|}
\hline & WoS subject category & $\begin{array}{l}\text { Scopus research areas } \\
\text { (categories/classification) }\end{array}$ \\
\hline 1 & Agriculture, multidisciplinary & Agriculture \\
\hline 2 & Biodiversity Conservation & Biodiversity and conservation \\
\hline 3 & Biology & Entomology \\
\hline 4 & Ecology & Environmental sciences and ecology \\
\hline 5 & Entomology & Forestry \\
\hline 6 & Environmental sciences & Plant sciences \\
\hline 7 & Environmental studies & Zoology \\
\hline 8 & Forestry & \\
\hline 9 & Horticulture & \\
\hline 10 & Multidisciplinary sciences & \\
\hline 11 & Mycology & \\
\hline 12 & Ornithology & \\
\hline 13 & Plant sciences & \\
\hline 14 & Soil science & \\
\hline 15 & Zoology & \\
\hline
\end{tabular}


(((forest* OR timber* OR tree* OR wood*) AND

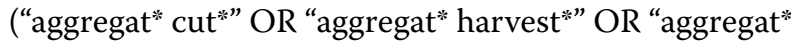
logging*" OR "alternative* cut*" OR "alternative* fell*" OR "alternative* harvest" "alternative* to clear* fell*" OR "alternative* to clearfell*" OR "alternative* to even age ${ }^{*}$ " OR "back" to nature*" OR "checker*"OR "close* to nature" ${ }^{*}$ " OR "common" sense $\mathrm{e}^{*}$ " OR "continuous* cover" ${ }^{*}$ " OR "continuous* forest" ${ }^{*}$ " OR "dauerwald" " OR "diversity* orient" OR "ecological" forestry" OR "ecological" silvicultur" OR "ecosystem* manag*" OR "gap* cut" OR "gap" fell"* OR "gap" harvest" " OR "gap* manag*" OR "gap* select*" OR "gap" silvicultur" OR "holistic*" OR "irregular" structure" ${ }^{*}$ " OR "irregular" OR "J shape ${ }^{*}$ " OR "low* impact" OR "multi" age" OR "multiage*" OR "multi" cohort" OR "multicohort" OR "multi" purpose $\mathrm{e}^{* \text { " }}$ OR "multipurpose $\mathrm{e}^{* \text { " }}$ OR "natural" disturbance ${ }^{*}$ base $e^{*}$ " OR "nature ${ }^{*}$ base $^{*}$ " OR "nature ${ }^{*}$ orient" ${ }^{*}$ OR "near" natural *" OR "new" forestry" OR "new* perspective" ${ }^{*}$ " OR "partial" cut" OR "partial" harvest*" OR "patch" cut" OR "patch" fell" OR "patch" harvest" OR "patch" logging" OR "permanent" forest" OR "plenter*" OR "polycyclic*" OR "positive* impact" OR "reduced" impact" OR "restoration* forest" OR "select" cut" OR "select" fell" OR "select" harvest" OR "select" logging" OR "select" manag*" OR "select" silvicultur" OR "single* tree" "strip" "OR "sustainable* forestry" OR "systemic* silvicultur" OR "systemic*" OR "target" diameter" "uneven" age" " OR "uneven" size ${ }^{* "}$ OR "low* impact" OR "common" sense" OR "dispers" cut" ${ }^{*}$ " OR "dispers* fell"*" OR "dispers* harvest" OR "dispers* silvicultur*") AND

("even age" " OR "clearcut" OR "clear" cut" OR "clear* fell" " OR "clearfell" OR "planted" forest" tion"

This search string is formatted for WoS and Scopus. For use in WoS 'TS =', and in Scopus 'TITLE-ABS-KEY' is added at the beginning of the search string.

Services other than WoS and Scopus are limited in functionality (e.g. search string length, no reference export). Therefore, we will use a simplified search strategy when using these other services. We will use most common and relevant search terms related to CCF (Table 2A). We will search with each CCF term individually or when the use of Boolean operators is possible combine the search with all relevant terms (Table 2A and B). We will screen the first 200 articles (sorted by relevance when possible) provided within each search. We will search for these terms in the title, abstract, keywords or anywhere in the article, depending on service capability. The details of these searches together with possible changes or addition of bibliographic databases, search engines and terms will be recorded. Applied document type restrictions will be recorded and reported in the

\section{Table 2 Terms used for simplified search}

A Search terms related to intervention: "continuous cover forestry",
"uneven-age forestry", "multiaged forestry","multi-aged
forestry", "multi-layered forestry", "partial harvest", "selective
logging"

review. Reference lists of relevant reviews and all eligible studies will also be systematically screened.

\section{Search update}

Once initial data extraction is done, before data synthesis, a search update will be conducted in Web of Science and Scopus. This search will be limited to start from the initial search. New articles will be screened and evaluated as before.

\section{Article screening and study eligibility criteria Screening process}

All results from searches will be added to an EndNote library and duplicates will be removed. Studies will be screened first by assessing the title. When the title indicates that relevant comparisons are potentially made, the paper will be considered relevant. If the title does not give a clear answer, the abstract is screened for relevance. Any study in which the title or abstract indicates that relevant comparison could have been made will be subjected to full-text screening. In the case of uncertainty, we will include the study and critically assess suitability by reading the full text. A second reviewer will evaluate a subset of studies from each screening stage independently. If disagreements arise regarding study relevance, discussions will be held to make a consensus decision. A list of rejected studies with reasons for exclusion from the full-text assessment will be provided in an additional file.

\section{Eligibility criteria}

Both peer-reviewed and grey literature will be considered. To be included, a study must meet the following criteria:

Eligible populations: Forests in the boreal and temperate vegetation zones.

Eligible intervention: Management systems that always maintain tree cover and use partial (selection) harvest to maintain at least two-age classes of production trees. Partial harvest can be divided into two main groups; group harvest and single-tree harvest. Group harvest, also called gap harvest or patch harvest is a harvesting method where smaller or larger groups of trees are harvested during felling. During single tree selection harvest, trees are chosen for felling individually, and larger canopy openings are created less likely. Both of the cuttings are 
used to achieve or maintain a desirable stand age/size structure (i.e. the ' $\mathrm{J}$ ' shape diameter curve with most small trees and decreasing number of large trees).

Harvest will be considered partial when $>30 \%$ of trees (based on reported measure, e.g. basal area, volume, biomass, canopy cover, stem density) are left after production harvesting. This threshold is subjective but not arbitrary as it is derived from the literature research with the threshold decidedly chosen in contrast to retention forestry. The threshold will be interpreted rigidly. Retention forestry or shelterwood harvest typically leaves $<30 \%$ of volume unharvested [4] and often do not result in multi-age structures throughout stand development. We will also include studies described as retention or shelterwood forestry provided that $>30 \%$ of trees are retained after harvest, and at least two age classes are achieved.

Ideally, comparisons with CC should be made between stands that already have uneven-aged structure and are then partially cut. This is often the case in observational studies. However, experiments typically start with a partial cut in the even-aged forest with the intention of converting them to the uneven-aged forest. If so, we will record this information as meta-data and consider it during analysis (i.e. pre-treatment forest condition as moderator). Since the effects of cutting on species are not dependent on the intention of the cutting (whether trees left will or will not be harvested in the future), we will consider all studies that compare clearcut to partial cut which meet the described eligibility criteria.

Eligible comparator: Clearcut, full stand harvested, no more than $5 \%$ trees retained (based on the reported measure). The threshold will be interpreted rigidly.

Eligible outcomes: Studies dealing with plants, animals and fungi. Most commonly reported measures of biodiversity that can be used for meta-analysis; species richness and abundance at stand scale. Other important measures of biodiversity (e.g. community composition, diversity indexes, the presence of forest interior or rare species) will be included in the narrative part of the review.

Eligible type of study design: Primary experimental and observational (e.g. chronosequence) field studies. We will consider before/after or control/intervention study designs and their combination. Model simulation studies, secondary research compilations and reviews will not be included.

Language: Full text written in English.

\section{Study validity assessment}

Studies that meet the eligibility criteria will be subjected to critical appraisal. We will assess their clarity and susceptibility to bias and categorise studies as having high or low validity (i.e. low or high risk of bias) relative to the review question. We will assess all forms of bias risk [17]. Studies that have any of the following limitations will be considered to have low validity and excluded.

1. No replication of interventions.

2. Intervention (CCF) and comparator (CC) sites not well matched (e.g. sites significantly different before harvesting).

3. Substantial confounding factors present (e.g. treatments carried out at significantly different times). Or additional treatments carried out at CCF and not at the CC sites that are not aspects commonly associated with each respective silvicultural approach (e.g. differences in grazing, burning).

4. Indication that outcomes in CCF vs CC sites were measured with potentially bias introducing methodology, differently or at significantly different times.

5. Some sites were excluded from the study after the treatment (e.g. unexpected disturbance in CCF but not in CC sites, insufficient time for fieldwork).

6. No or partial reporting of measurements indicated in the methods.

The two first conditions deal with susceptibility to selection bias, the third one with performance bias, fourth with detection bias, fifth with attrition bias and sixth with reporting bias, according to CEE guidelines [17]. Other types of bias risk not classifiable under the above categories (e.g. funding source) will also be considered.

Studies will also be excluded if the information regarding these issues is unclear and the validity of the study cannot be assessed. Specifically, we will also exclude studies due to the following:

1. Methodology not sufficiently described.

2. Data cannot be interpreted.

Studies that do not have any of those limitations will be considered to have high validity and included in the review.

The process of critical appraisal will be transparently documented. A list of excluded articles, including reasons for exclusion, will be recorded and presented in an additional file. Study quality and generalisability will be assessed by one reviewer. Doubtful cases will be discussed in the review team. Additional criteria or modification for critical appraisal will be done if necessary and documented. 


\section{Data coding and extraction strategy}

Data on species richness and abundance will be extracted from tables. This data will include means, a measure of variation (standard deviation, standard error, confidence interval, etc.) and sample size. All measures of variation will be converted to standard deviation. If data is not presented in tables but in figures, we will contact the corresponding author and ask for the data. If authors do not provide the data, we will extract it from figures using WebPlotDigitizer [21]. Authors will also be contacted if relevant data is not presented but is likely to be available (i.e. used for more advanced analysis). If raw data is provided, the necessary summary data will be calculated. When data cannot be extracted from the paper, and the authors do not provide it, we will use the study in the qualitative part of the review.

Studies that do not report species richness and/or abundance but report other types of biodiversity assessment (e.g. community composition, diversity indexes, the presence of forest interior or rare species) will also be used in the qualitative part of the review. From these studies, text describing relevant comparison will be extracted and stored in a spreadsheet.

If possible, species will be separated into forest, open habitat and generalist species. This is a key aspect when evaluating habitat suitability and interpreting management effects on biodiversity.

In the case of uncertainty regarding reported meta-data or data in papers, we will contact corresponding authors for clarification. If the authors do not respond, or cannot provide sufficient explanation for the issues raised, the paper will not be used in the review. This process will be documented and presented in an additional file.

Associated meta-data together with potential effect modifiers will be extracted and coded as follows.

1. Unique study ID

2. Source

1. Bibliographic database (1-7)

2. Search engine $(1,2)$

3. Specialist website

4. ...

3. Author(s)

4. Year

5. Title

6. Journal

7. DOI

8. Inclusion/exclusion (according to eligibility criteria)

1. Excluded (low validity or unclear description)

2. Included (high validity)
9. Reason for exclusion (bias type)

10. Study type

1. Observational

2. Experiment

3. ...

11. \% of cut based on
1. Volume
2. Basal area
3. Crown cover
4. No of stems
5. ...

12. Variance reported

1. No

2. Yes

13. Part of a large experiment

1. No

2. If yes then name of the experiment

14. Control available

1. No

2. Yes

15. Biome (temperate or boreal)

16. Location coordinates

17. Location description (landscape context)

18. Country

19. Elevation

20. Climate

21. Forest type

22. Time after treatment the study was made

23. Tree species composition

24. Spatial scale (stand or landscape)

25. Pre-cut forest state

26. Pre-cut forest age

27. Year of cutting

28. Interventions (details about harvesting)

29. CC logging (\% of retained trees)

30. CCF logging type (e.g. group or single tree harvest)

31. Removal \%

32. Logging type CCF2 (typically several types of partial cuts are performed)

33. Removal \%

34. Type of sampling methodology used

This data will be made available in an additional file. The main reviewer will extract data and meta-data and record the process transparently according to the strategy. A second reviewer will check the extracted data and edits will be made if necessary. 
Potential effect modifiers and reasons for heterogeneity Depending on the availability of data provided in the papers, we will extract various potential effect modifiers according to the above list. These modifiers were chosen because they have potential effects on how harvesting influences biodiversity. The list is not exhaustive, and other effect modifiers and causes of heterogeneity will be considered during the review process.

\section{Data synthesis and presentation}

A narrative synthesis of all included studies that describes the quality of the results and study findings will be written. Tables with summarised results will be prepared. Scoping work indicates that enough data will be available to also perform quantitative analysis. For this, we will follow guidelines and recommendations from literature [22-25].

Standardized mean difference in the form of Hedges' g will be calculated with accompanying variance estimates for species richness and abundance. If included studies do not report variance estimates, but the share of these studies is not too large $(<20 \%)$, we will impute the missing values [26]. Imputation methods will depend on the characteristics of missing data.

While exact details of quantitative analysis depend on the extracted data, we propose a comprehensive synthesis and presentation strategy. Effect sizes will be explored using various methods including randomeffects models, subgroup analysis and meta-regression. Due to the nature of the study topic, many effect sizes will be non-independent. Data from many species across many years is typically collected during observations or after experiments. To account for this, we will include a study as a random variable in the models. The effect of different moderators will be tested to find out what best explains the observed patterns (e.g. heterogeneity) in data. Generally, we will include a moderator if at least ten effect sizes per group are available. Correlation between moderators (i.e. multicollinearity) will also be considered.

To explore heterogeneity (inconsistency among studies), we will also analyse the effect sizes of different data subsets (i.e. taxonomic groups, forest type and habitat preference groups if possible). Although this is similar to meta-regression, it allows evaluation of heterogeneity, and the calculation of different heterogeneity statistics, $\left(\tau^{2}, \mathrm{Q}\right.$ and $\left.I^{2}\right)$ for different groups of interest.

The landscape context, time after treatment, forest type and other effect modifiers are expected to have significant influence on the results and will likely explain important aspects of the effects. Therefore, we will explore the influence of various effect modifiers and document this process. The results will be presented in tables and figures.

There is no 'best' way to estimate the robustness of the results and a variety of methods should be used [27]. We will perform sensitivity analysis by using several methods including leave-one-out meta-analysis, running the analysis with and without influential studies (effect sizes), running models with and without non-independence and comparing model fit statistics. If data imputation will be performed, we will perform and report analysis separately for all data, for data excluding studies with imputed data, and for all data using unweighted meta-analysis. This will also serve as a part of the sensitivity analysis. We will assess the risk of publication bias by fail-safe $\mathrm{N}$ and funnel plots using the trim and fill function. Significant knowledge gaps will be described and discussed in terms of future research needs.

\section{Additional files}

Additional file 1. ROSES form

Additional file 2. Systematically extracted terms used to describe CCF and associated harvesting methods.

Authors' contributions

The manuscript was drafted by MS. AF and ML edited the manuscript. All authors read and approved the final manuscript.

\section{Acknowledgements \\ We are grateful for CEE community for providing useful and clear guidelines to prepare high-quality comprehensive manuscripts. We are also thankful to the} reviewers for their thorough revisions of our protocol.

\section{Competing interests}

The authors declare that they have no competing interests. Members of the review team have not authored any publications that meet the inclusion criteria of the proposed review.

Availability of data and materials Not applicable.

\section{Consent for publication}

Not applicable.

Ethics approval and consent to participate Not applicable.

\section{Funding}

Funding for this study was provided by Southern Swedish Forest Research Centre, The Swedish University of Agricultural Sciences, SLU. Special thanks are due to the European Union for partial support of this protocol preparation through funding of the ALTERFOR project within the Horizon 2020 research and innovation programme under grant agreement No 676754.

\section{Publisher's Note}

Springer Nature remains neutral with regard to jurisdictional claims in published maps and institutional affiliations. 
Received: 1 June 2018 Accepted: 19 November 2018

Published online: 06 December 2018

\section{References}

1. Duncker PS, Raulund-Rasmussen K, Gundersen P, Katzensteiner K, De Jong J, Ravn HP, Smith M, Eckmüllner O, Spiecker H. How forest management affects ecosystem services, including timber production and economic return: synergies and trade-offs. Ecol Soc. 2012. https://doi. org/10.5751/ES-05066-170450.

2. MEA. Millenium ecosystem assessment: ecosystems and human wellbeing: biodiversity synthesis. Washington, D.C.: World Resources Institute; 2005. p. 1-85.

3. Lindenmayer BD, Franklin JF. Conserving forest biodiversity: a comprehensive multiscaled approach. Washington: Island Press; 2002.

4. Gustafsson L, Baker SC, Bauhus J, Beese WJ, Brodie A, Kouki J, Lindenmayer DB, Lohmus A, Martinez Pastur G, Messier C, et al. Retention forestry to maintain multifunctional forests: a world perspective. Bioscience. 2012;62(7):633-45.

5. Kuuluvainen T. Forest management and biodiversity conservation based on natural ecosystem dynamics in northern Europe: the complexity challenge. Ambio. 2009;38(6):309-15.

6. Angelstam PK. Maintaining and restoring biodiversity in European boreal forests by developing natural disturbance regimes. J Veg Sci. 1998;9(4):593-602.

7. Kuuluvainen T, Tahvonen O, Aakala T. Even-aged and uneven-aged forest management in boreal Fennoscandia: a review. Ambio. 2012;41(7):720-37.

8. Lindenmayer DB, Franklin JF, Fischer J. General management principles and a checklist of strategies to guide forest biodiversity conservation. Biol Conserv. 2006;131(3):433-45.

9. Fedrowitz K, Koricheva J, Baker DC, Lindenmayer DB, Palik B, Rosenvald R, Beese W, Franklin JF, Kouki J, Macdonald E. Can retention forestry help conserve biodiversity? A meta-analysis. J Appl Ecol. 2014;51(6):1669-79.

10. Mori AS, Kitagawa R. Retention forestry as a major paradigm for safeguarding forest biodiversity in productive landscapes: a global metaanalysis. Biol Conserv. 2014;175:65-73.

11. Pommerening A, Murphy ST. A review of the history, definitions and methods of continuous cover forestry with special attention to afforestation and restocking. Forestry. 2004;77(1):27-44

12. Kuuluvainen T, Wallenius TH, Kauhanen H, Aakala T, Mikkola K, Demidova N, Ogibin B. Episodic, patchy disturbances characterize an old-growth
Picea abies dominated forest landscape in northeastern Europe. For Ecol Manag. 2014;320:96-103.

13. United Nations. The convention on biological diversity. 1992

14. Nolet P, Kneeshaw D, Messier C, Beland M. Comparing the effects of evenand uneven-aged silviculture on ecological diversity and processes: a review. Ecol Evol. 2018;8(2):1217-26.

15. Schall P, Gossner MM, Heinrichs S, Fischer M, Boch S, Prati D, Jung K, Baumgartner $\mathrm{V}$, Blaser S, Bohm S, et al. The impact of even-aged and uneven-aged forest management on regional biodiversity of multiple taxa in European beech forests. J Appl Ecol. 2018;55(1):267-78.

16. Peura M, Burgas D, Eyvindson K, Repo A, Mönkkönen M. Continuous cover forestry is a cost-efficient tool to increase multifunctionality of boreal production forests in Fennoscandia. Biol Conserv. 2018:217:104-12

17. Collaboration for Environmental Evidence. 2018. Guidelines and Standards for Evidence synthesis in Environmental Management. Version 5.0 (AS Pullin, GK Frampton, B Livoreil \& G Petrokofsky, Eds). http://www.envir onmentalevidence.org/information-for-authors Accessed 3 Apr 2018.

18. Haddaway N, Macura B, Whaley P, Pullin A. ROSES for systematic review protocols. Version 1.0. 2017

19. O'Hara KL. Multiaged silviculture: managing for complex forest stand structures. New York: Oxford Univ Press; 2014.

20. Puettmann KJ, Wilson SM, Baker SC, Donoso PJ, Drössler L, Amente G, Harvey BD, Knoke T, Lu Y, Nocentini S, et al. Silvicultural alternatives to conventional even-aged forest management-what limits global adoption? For Ecosyst. 2015;2(1):1-8.

21. Rohatgi A. WebPlotDigitizer. 2018. https://automeris.io/WebPlotDigitizer. Accessed 3 Apr 2018.

22. Koricheva J, Gurevitch J. Uses and misuses of meta-analysis in plant ecology. J Ecol. 2014;102(4):828-44.

23. Nakagawa S, Santos ESA. Methodological issues and advances in biological meta-analysis. Evol Ecol. 2012;26(5):1253-74.

24. Noble DWA, Lagisz M, O'dea RE, Nakagawa S. Nonindependence and sensitivity analyses in ecological and evolutionary meta-analyses. Mol Ecol. 2017;26(9):2410-25.

25. Viechtbauer W. Conducting meta-analyses in $\mathrm{R}$ with the metafor package J Stat Softw. 2010;36(3):1-48.

26. Ellington EH, Bastille-Rousseau G, Austin C, Landolt KN, Pond BA, Rees EE, Robar N, Murray DL. Using multiple imputation to estimate missing data in meta-regression. Methods Ecol Evol. 2015;6(2):153-63.

27. Nakagawa S, Noble DWA, Senior AM, Lagisz M. Meta-evaluation of metaanalysis: ten appraisal questions for biologists. BMC Biol. 2017:15:18.
Ready to submit your research? Choose BMC and benefit from:

- fast, convenient online submission

- thorough peer review by experienced researchers in your field

- rapid publication on acceptance

- support for research data, including large and complex data types

- gold Open Access which fosters wider collaboration and increased citations

- maximum visibility for your research: over 100M website views per year

At BMC, research is always in progress.

Learn more biomedcentral.com/submissions 